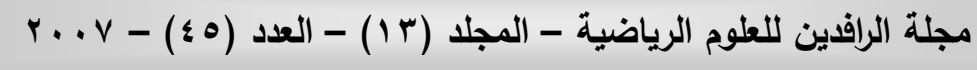

\section{تقويم التركيبات الهجومية المركبة أكثر تأثيرا و استخداما بالكرة الطائرة \\ م.د.أزاد حسن قادر}

جامعة صلاح الدين /كلية التربية الرياضية

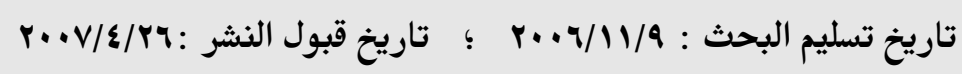

الملخص

هاف البحث الى :

التعرف على مركبات اللعب الهجومي الاكثر تأثثراً و استخداماً لدى لاعبي فرق

منتخبات الجامعات العراقية بالكرة الطائرة.

وتكمن اهمية البحث في الكثف او التعرف على ناثير مركبات اللعب الهجومي ، وأيهما

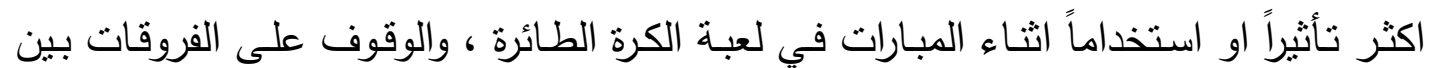
مستويات المركبات الهجومية المركبة.

وقد ثبلورت مشكلة البحث في عدم مقدرة الفريق المهاجم على اللعب بانماط هجومية مختلفة وعدم إجادته للمركبات الهجومية من ناحية الأداء الفني.

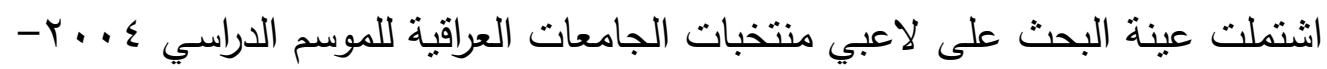

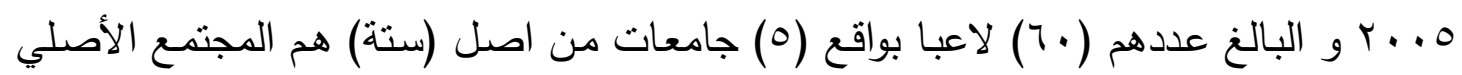
للبحث ، و بهذا فأن نسبة عينة البحث قد بلغت (r/\%) من المجتمع الاصلي ، وقد نم استخدام

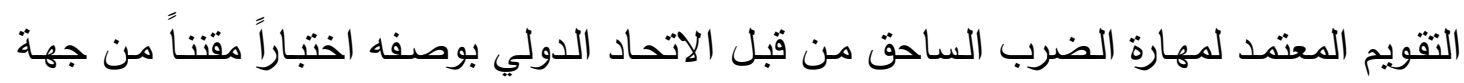
معتمدة.

وكان منهج البحث المستخدم هو المنهج الوصفي بالاسلوب المسحي لملائمة مشكلة

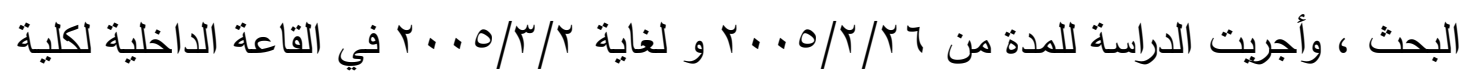
التربية الرياضية في جامعة صلاح الدين - اربيل. 


$$
\begin{aligned}
& \text { استتتج الباحث عدد من الاستتتاجات كان اهمها : } \\
& \text { ان مركب اللعب الهجومي العالي و السريع اكثر استخداماً و تأثيراً في حين. } \\
& \text { ا - ان مركب اللعب الهجومي في المنطقة الخلفية و اللعب المتوسط الارتفاع اقل استخداماً و } \\
& \text { تأثنرا لاًى عينة البحث. } \\
& \text { وق قدم الباحث عدة توصيات الهمها : } \\
& \text { أ/ ضـرورة مراعـات مبـدأ التخصص و اختيـار لاعبـين متخصـصين لانـواع مركبـات اللعـب } \\
& \text { الهجومي على الثبكة فعلا. } \\
& \text { ب/ الاهتمـام بتدريب انواع متعددة من المركبات ضدمن الوحدة التدريبية و التدريب على اداء } \\
& \text { مركبات هجومية في المنطقة الخلفية لآهميتها الحاضرة في مباريات الكرة الطائرة. } \\
& \text { ABSTRACT }
\end{aligned}
$$

A study of offensive component compositions of most effective and used in volleyball.

\author{
Dr. Azad H.Qadir
}

University of salahaddin / college of physical education

\begin{abstract}
Important of the study :
The important of this research was to discover and identify the offensive component compositions, and which is the most effective and useable during the match of volley ball Also, to know the differences between the levels of offensive component compositions.
\end{abstract}

\title{
Statement of the problem :
}

The major problem of the research was the following :

- to was the following the offensive team has no ability to play with different offensive compositions in a technical performance.

Aim of the study:

1-To identify on offensive component compositions of most effective and used of Iraqi varsity teams of universities in volley ball.

\section{Methodology}

- A sample of (60) players of Iraqi university varsity teams of 2004-2005.

- A sample total of (5) from (6) university was conducted and the percentage of the sample was $(83 \%)$.

- A descriptive research was conducted in this study for the time of $26 / 2 / 2005-2 / 3 / 2005$ at the college of physical education, university of salahaddin. 
- The researcher used standard test for the evaluator of the skill of spiking sere which has been used by international federation as an accurate instrument test :

\section{Results and Recommmmedations.}

On the basis of the basis of the analysis of the data, the following conclusions were presented:

1. The high and fast offensive Components of the game was most used and affective. On the other hand, the medium offensive Components of the back ward zone.

2. More emphasis on specializing and selecting players of all affective Components should be taken into consideration.

3. More emphasis on training of all kind of Components on the training unit should be taken into consideration especially on the back ward zone of the volleyball.
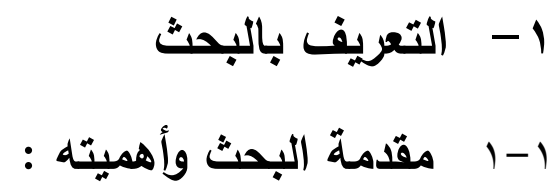

مما لاثك فيه ان ارتفاع مستوى التقدم الرياضي المعاصر ولاسيما في الحقبة الاخيرة

مـن القرن الماضـي كـان هدفا يسعى اليهـ الكثير من العلمـاء و المتخصصين في المجـال الرياضي عامة ومجال الالعاب وبعض الانثطة الرياضية المتميزة خاصة.

فمنهم من سعى الى تقديم المعارف و المعلومات بصورة مبسطة لمدربهم بهدف الاعداد

و التتمية لبلوغ المستويات العالية. و الكرة الطائرة شكلا من انكال العاب الكرة واحد الانشطة الرياضية ذات الطبيعة الخاصة التي تتميز بحدة الآثارة و يتجلى ذلك من خلاد التشكيلات

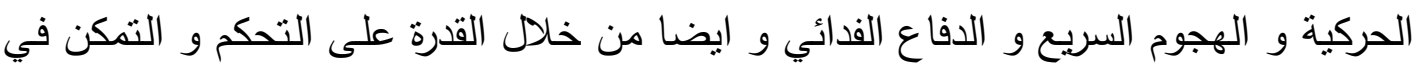

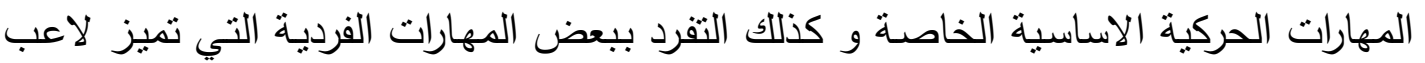
عن اخر في ضوء ما يتمتع به من حصائص (')

ومن هنا تأني أهمية البحث في الكثف او التعرف على نأثير مركبات اللعب الهجومي ، و ايهما اكثر استخداماً اثثاء المباراة في لعبة الكرة الطائرة.

(1) زكي محمد محمد حسن : الكرة الطائرة تقنيات حديثة في التعليم و التدريب ، ملتقى الفكر ، اسكندرية ، مصر ، ب ت صك صندا. 
للهجوم او العمل الهجومي فلسفة خاصـة شانه في ذلك شـان اى شىء لـه فلسفة فهناك فلسفة في اتقان المهارات الأساسية و تعد مهارة الضرب الساحق من المهارات الهجومية

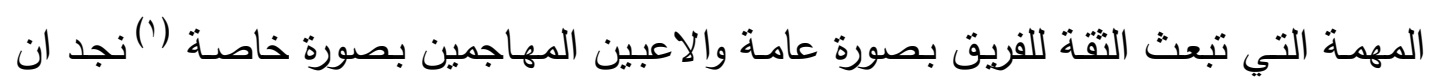

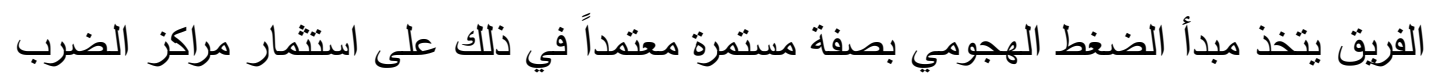
الامامية الهجومية يساعد في ذلك الهروب من حائط الصدد المكون امام هذا الهجوم.

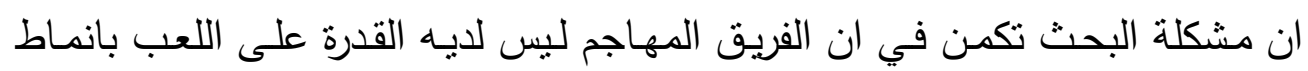
اللعب الهجومية المختلفة و عدم اجادته للمركبات الهجومية و هذه المشكلة تؤدي الى توتر

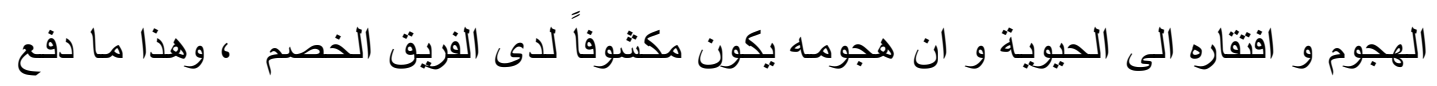
الباحث الى دراسة هذا الموضوع.

$$
\begin{aligned}
& \text { 1- } \\
& \text { يهذف البحث الى :- }
\end{aligned}
$$

1-r-1 التعرف على مركبات اللعب الهجومي الاكثر تأثنراً للدى لاعبي فرق منتخبات

الجامعات العراقية بالكرة الطائرة .

ا I-r-r-r معرفة المركبات الاكثر استخداماً لاى افراد العينة.

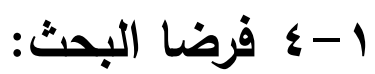

1-§-1 ان مركب اللعب الهجومي السريع و اللعب العالي اكثر تأثثر و استخداما لدى عينة

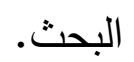

1-؟-r ان مركب اللعب الهجومي في المنطقة الخلفية و متوسط الارتفاع اقل استخداما و

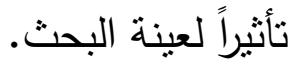

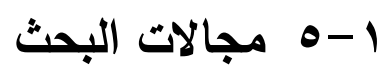

1-0-1- المجال البشري : ل لاعبو فرق منتخبات الجامعات العراقية بالكرة الطائرة .

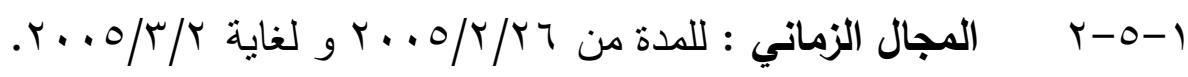

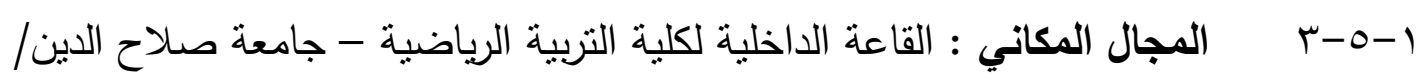
اربيل.

\section{r - الاراسات النظرية}

(1 ) ازاد حسن قادر : دراسة مقارنة لتأثير مركبات العب الهجومي في المنطقة الامامية و الخلفية على نتيجة

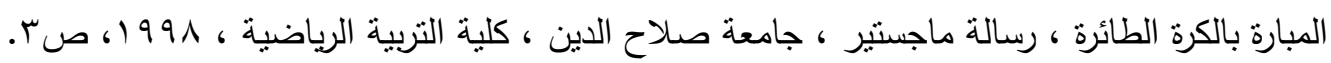




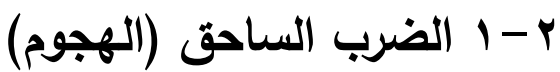

يعد الهجوم في الكرة الطائرة أحد السمات او الخصائص التي تميز اي فريق كرة طائرة

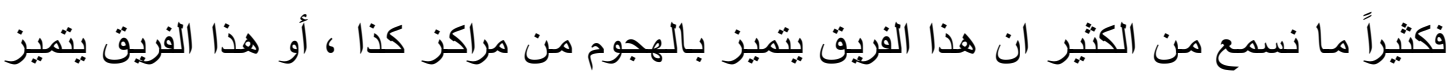

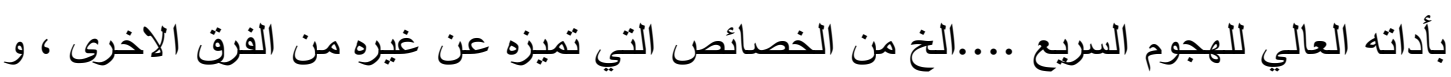

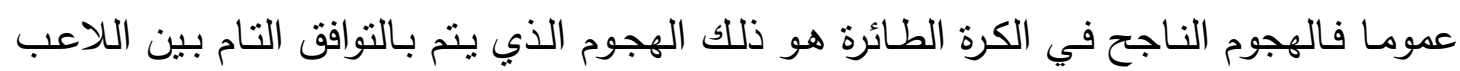

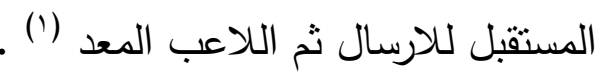

الضرب الساحق من اهم طرائق الهجوم المستخدمة من جانب اللاعبين من مباريات

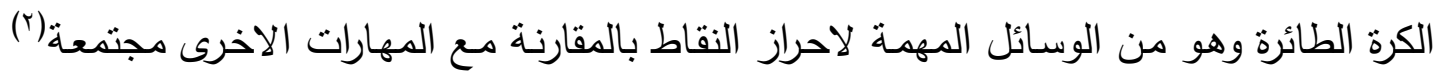

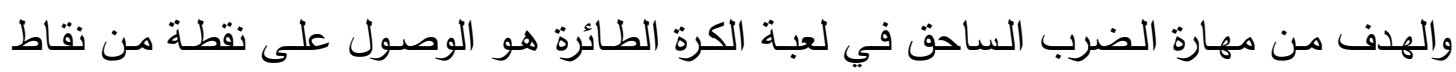

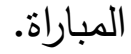

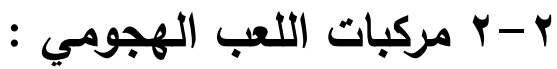

ان مهارة الضرب الساحق تصنف حسب منـاطق اللعب الى صنفين هــا الضرب الساحق من المنطقة الامامية و المنطقة الخلفية ـ و يمكن تصنيف الضربات الساحقة حسب

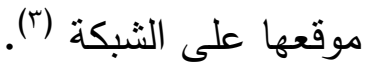

وتقسم مركبات اللعب الهجومي السريع الى انواع الاتية كما موضع في الثكل رقم (1).

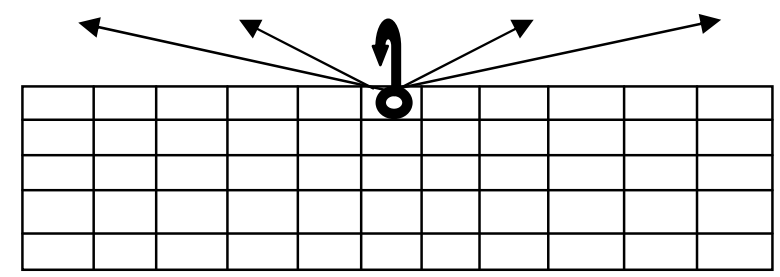

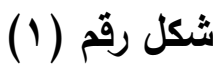

يوضح مركبات اللعب الهجومي السريع

(A-Quick )

- مركبات اللعب الهجومي سريع القصير

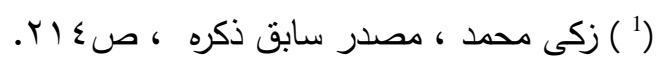

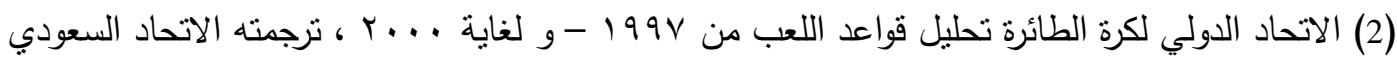

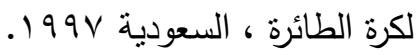

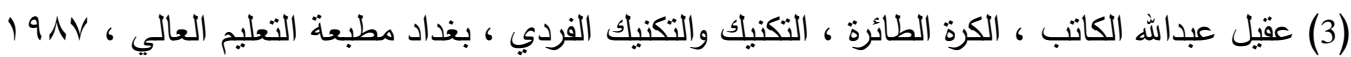


(B-Quick and c-Quick) مركبات اللعب الهجومي سريع المتوسط (D-Quick ) - مركبات اللعب الهجومي سريع الطويل r - r مركبات اللعب الهجومي متوسط الأرتفاع

هذا النوع من المركبات تشبه الى حد بعيد مركبات اللعب الهجومي السريع الطويل ، الا

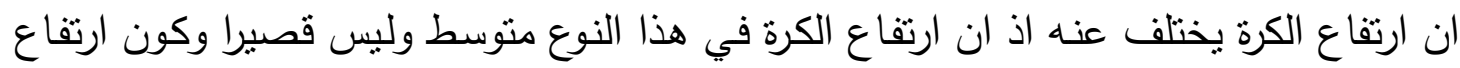

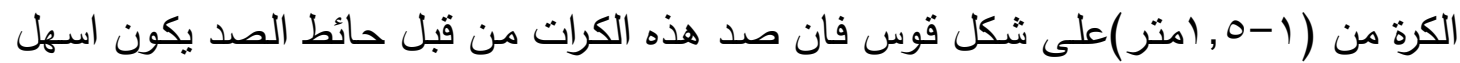

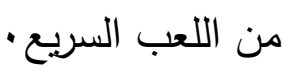

\section{r - ب مركبات اللعب الهجومي عالية الأرتفاع}

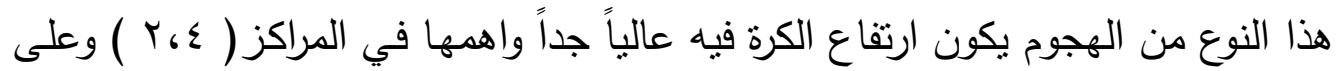

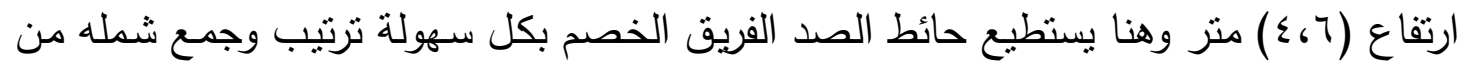

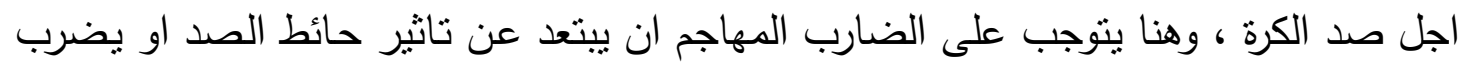

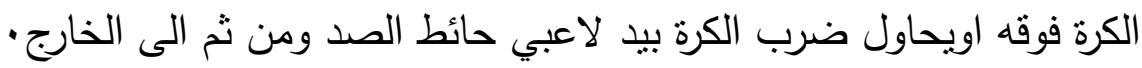

r-ه مركبات اللعب الهجومي في المنطقة الخلفية

ان مركبات اللعب في المنطقة الخلفية كانت تستعمل بشكل عشوائي وفي الضرورات الملحة من اللاعبين لكنها دخلت بشكل علمي ضـن هذه المركبات عندما جـاء البولونيون

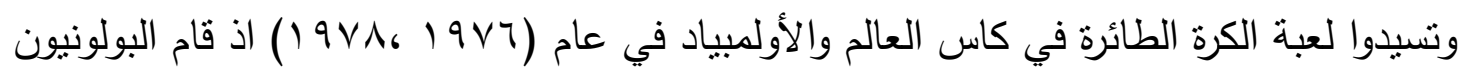
بمركبات اللعب من الخلف وفاجأوا العالم بهذه الطريقة المتعة ومنذ ذلك الوقت تطورت مركبات اللعب من الخلف واصبحت جزأ لايتجزاء من المركبات الهجومية الأخرى.

\section{ب- منهج البحث و اجراعاته الميداتية : ب}

المنهج هو الطريقة التي يتبعها الباحث في دراسته لحل للمشكلة ولاكتشاف الحقيقة

وقد استخدم الباحث المنهج الوصفي باسلوب المسح لملائمة مشكلة البحث. r-r

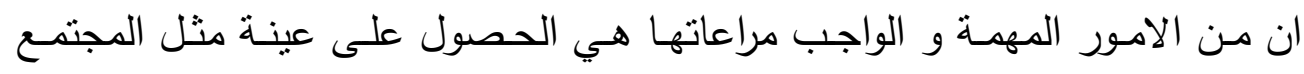

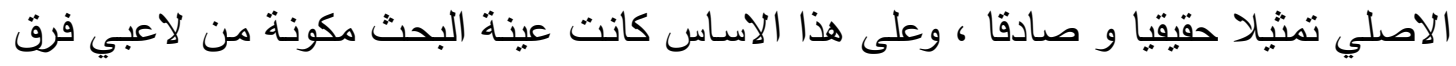

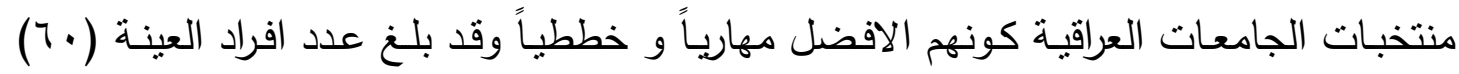
لاعباً و الذين ممنلو خمس الجامعات من مجموع ست وهم (صلاح الدين ، السليمانية ، بغداد ، 
القادية ، الموصل ) بعد انسحاب جامعة تكريت من لعب المرحلة النهائية من الدوري و بهذا عينة البحث مثلت (r^ \% \%) من مجتمع الاصل. r-r

من اجل التوصل الى الحقائق فقد استعان الباحث بأدوات تتماثـى وطبيعة المشكلة و

$$
\begin{aligned}
& \text { البيانات و المعلومات التي تتطلبها ، وهذه الادوات هي:- } \\
& \text { المصادر العربية و الاجنبية. }
\end{aligned}
$$

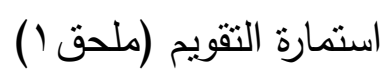

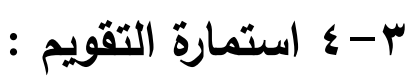

تم استخدام درجات لتقويم مهارة الضرب الساحق المعتمدة من اللجنـة الفنية التابعة

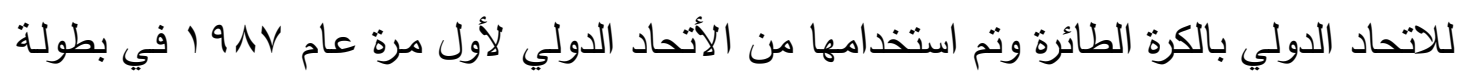

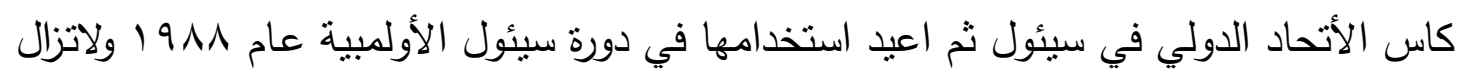

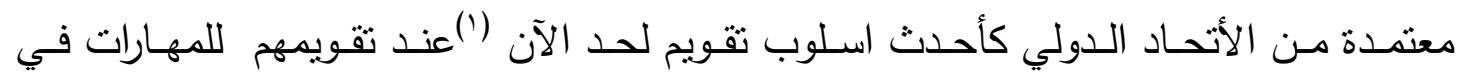
البطولات الدولية وهي بذلك اكتسبت صفة الصدق واثبات و الموضوعية ولآنها مقننة ودرجات التقويم تعطى كالآتي لجميع انواع الهجوم بالكرة الطائرة : 1- الضرب الساحق بسجل نقطة من اجرائه يعطى (؟ّ) درجات.

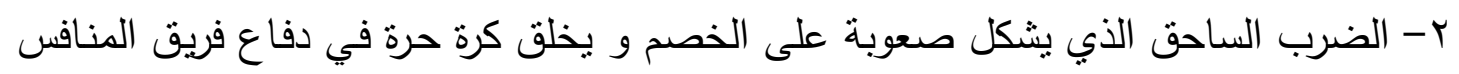

$$
\text { يعطى (r) (Y) درجة. }
$$

צ- الضرب الساحق الذي ينتج عن دفاع جيد من قبل الخصم يعطى (1) درجة. ع- الضرب الساحق الفاشل يعطى ( •) درجة. ب- ب- الوسائل الإحصائية :

(1)

$$
\begin{aligned}
& \text { تم استخدام الوسائل الاحصائية التالية (ل) } \\
& \text { 1. النسبة المئوية = (الجزء/ الكل)××..1 } \\
& \text { r. الوسط الحسابي = مج س/ن } \\
& \text { צ- عرض و مناقشة النتائج }
\end{aligned}
$$

ع - 1 عرض و تحليل تقويم محاولات الهجوم المركبة و استخدامها ـ

(1) Back, horst, Technical report federation international volleyball, 1998.

(') عبدالرحمن عس، مبادى الاحصاء في التربية وعلم النفس ، الاردن ، مكتبة الاقصى ، 1911 ، صبr 198 
يتضمن هذا الباب عرض و مناقشة الننائج التي توصل اليها الباحث ، وتم درجها في

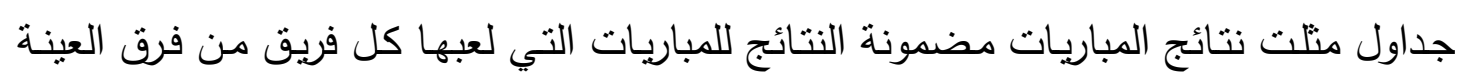
وذلك اختصاراً للجداول والاثشال و لتكن مقارنتها اسهل للقارىه.

؛ - 1 - 1 عرض وتحليل و تقويم نتائج المحاولات المستخدمة للهجوم المركب لافراد

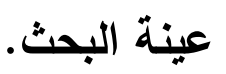

يبين الجدول رقم ( ) عدد المحاولات الكلية للهجوم المركب فبالنسبة لجامعة صـاح

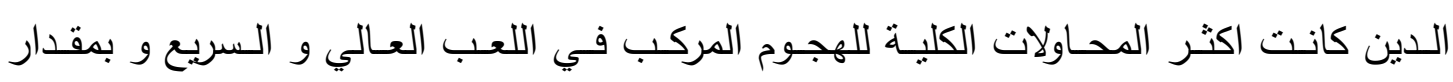

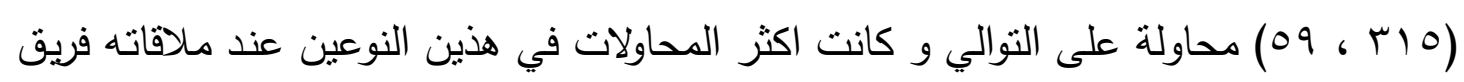

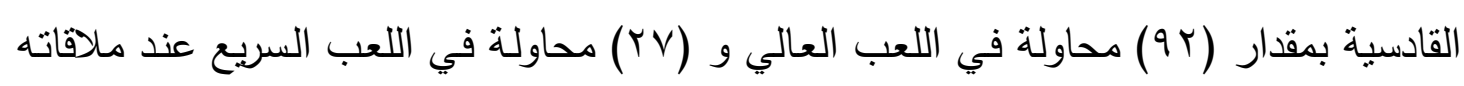

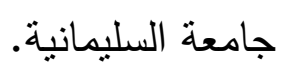

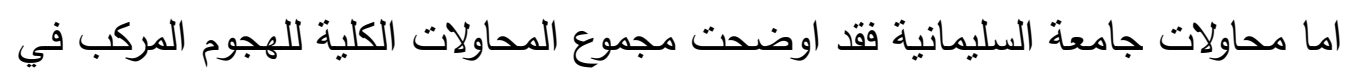

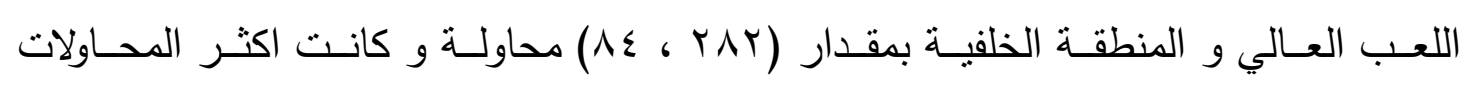

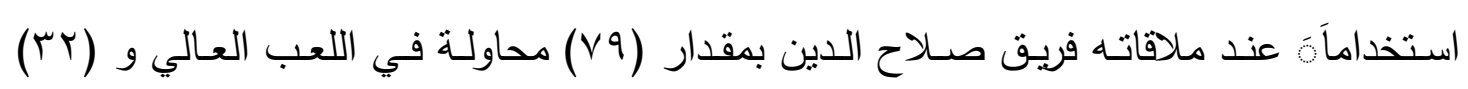
محاولة في المنطقة الخلفية عند ملاقاته جامعة بغداد.

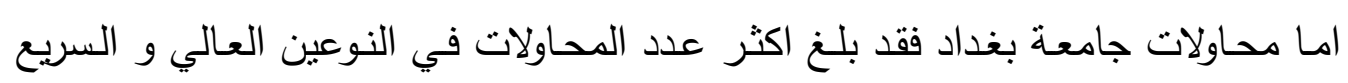

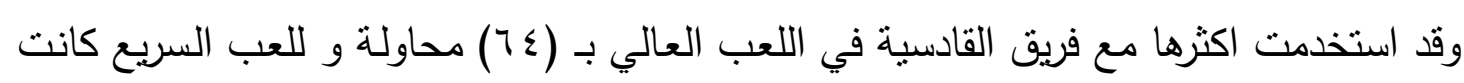

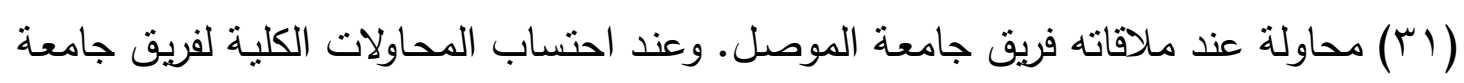

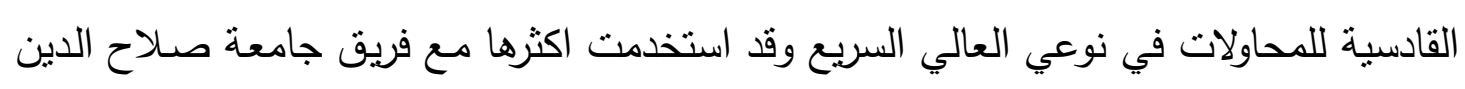
في اللعب العالي بمجموع (Y (Y) محاولة واللعب السريع كانت (Y) (Y) محاولة.

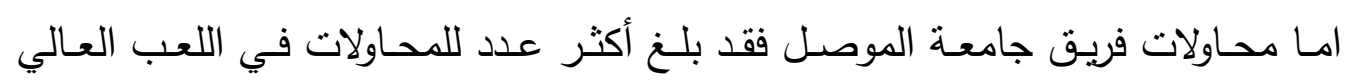

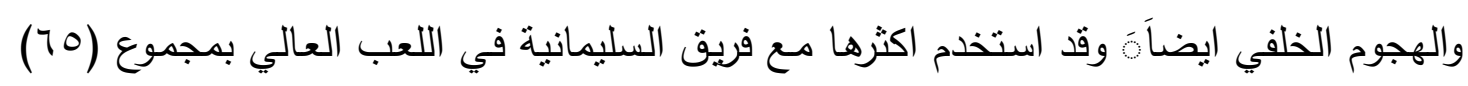

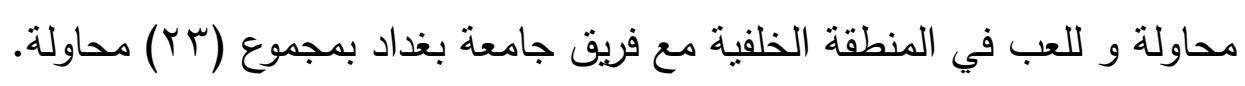

وبعد احتساب الوسط الحسابي لعدد المحاولات الكلية و لجميع مباريات عينة البحث

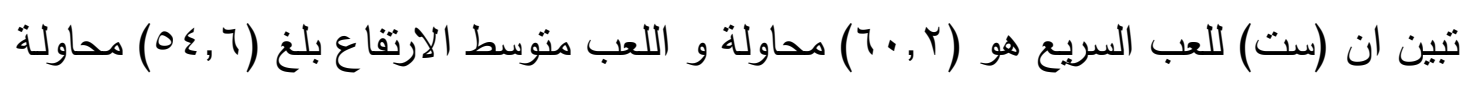

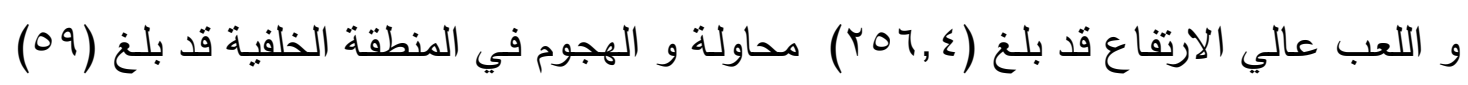
محاولة.

اما الجدول رقم (ץ) فأنه يبين نتائج النسب المئوية للمركبات الهجومية المؤثرة ، فكانت

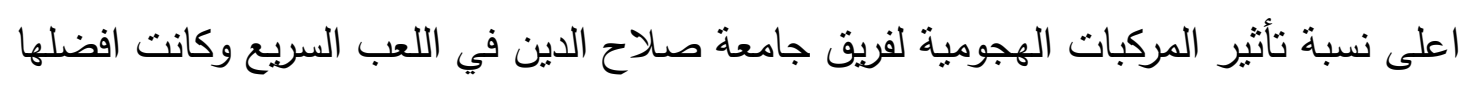


عند مباراته مع فريق جامعة الموصل بمقدار (0 . به \% \%) واحتل اللعب العالي المرتبة الثانية

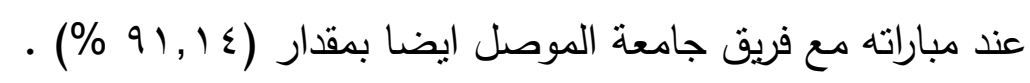

وعند احتساب الوسط الحسابي للنسب المئوية لفريق جامعة صلاح الدين فكانت افضلها

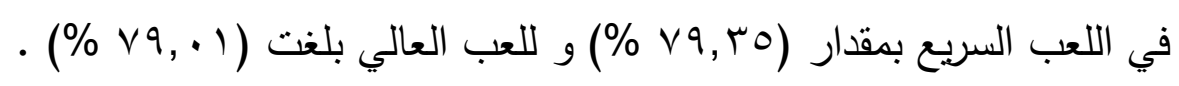

اما الوسط الحسابي لنتائج فريق جامعة السليمانية فقد بلغت في اللعب السريع بنسبة

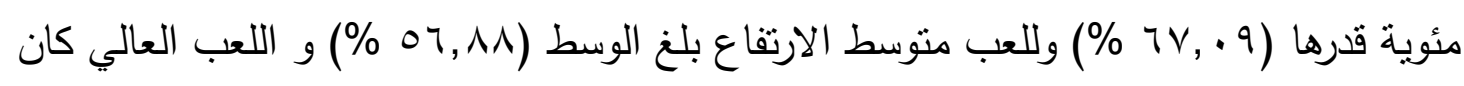

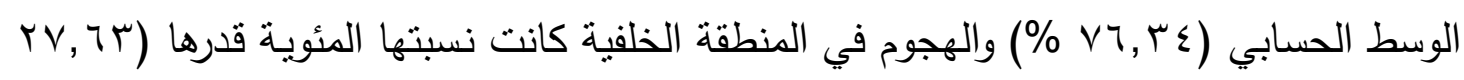

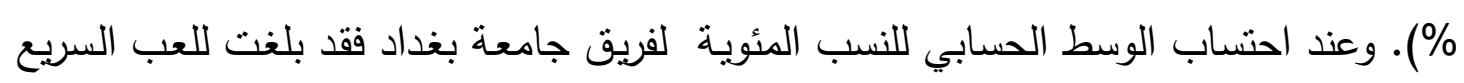

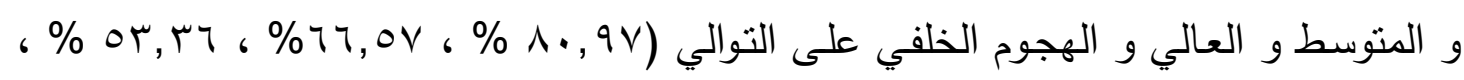

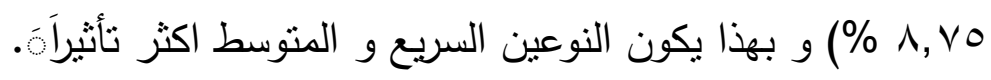

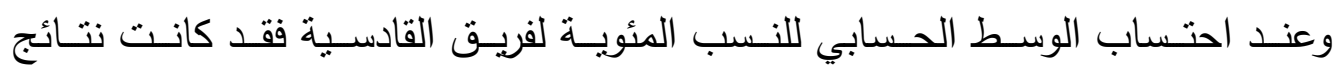

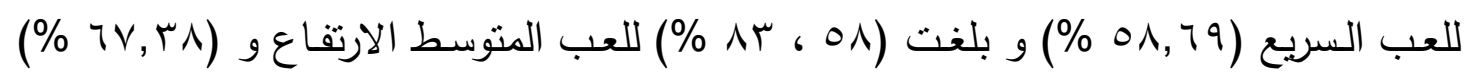

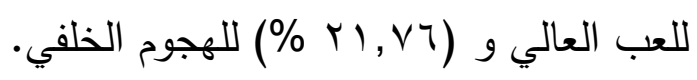

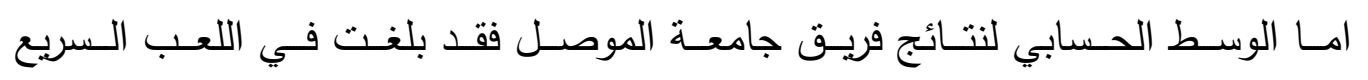

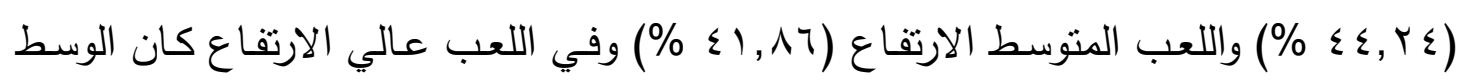

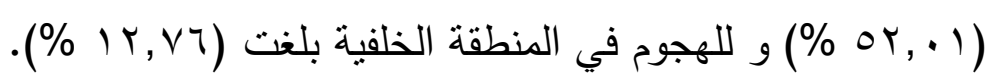

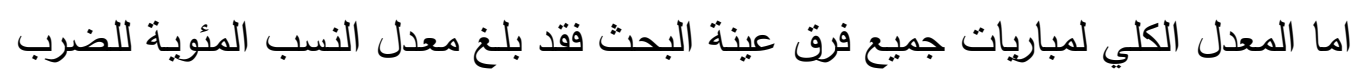

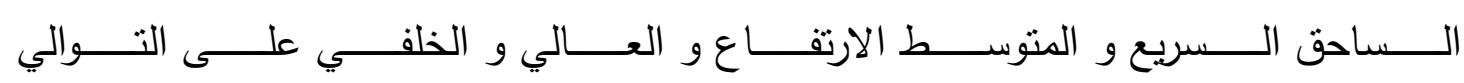

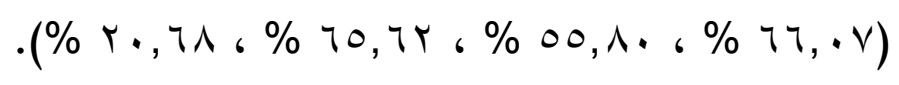




\section{جدول رقم ( ) يبين عدد محاولات مركبات الهجوم لفرق عينة البحث}

\begin{tabular}{|c|c|c|c|c|c|c|c|c|c|c|c|c|c|c|c|c|c|c|c|c|c|c|c|c|}
\hline \multicolumn{4}{|c|}{ المحاولات الكلية للهجوم } & \multicolumn{4}{|c|}{ 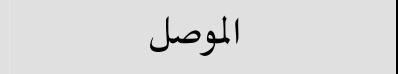 } & \multicolumn{4}{|c|}{ 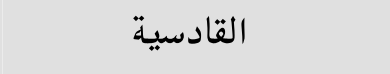 } & \multicolumn{4}{|c|}{ 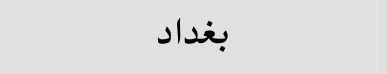 } & \multicolumn{4}{|c|}{ السليمانية } & \multicolumn{4}{|c|}{ صلاح الدين } & \multirow{2}{*}{ المركبات الفرق } \\
\hline 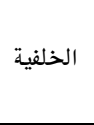 & عالية & 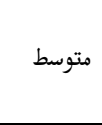 & 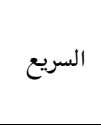 & الخلفية & 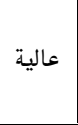 & متوسط & 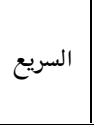 & 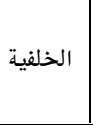 & عالية & 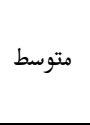 & 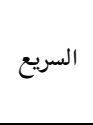 & الخلفية & عالية & متوسط & 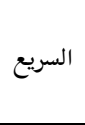 & الخلفية & 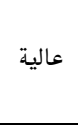 & متوسط & 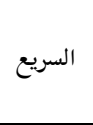 & الخلفية & عالارتغاع & متلورسط & السريع & \\
\hline 00 & Mlo & ro & 89 & $\cdot r$ & 71 & $\cdot 0$ & $1 \varepsilon$ & ir & 94 & 10 & $\cdot 1$ & r) & V乏 & 11 & $1 \cdot$ & 11 & $\wedge 1$ & rr & rV & $=$ & $=$ & $=$ & $=$ & صلاح الدين \\
\hline$\wedge \varepsilon$ & rAY & vo & $0 \wedge$ & $1 \varepsilon$ & 01 & ri & 11 & IV & VV & .9 & .9 & rr & $\uparrow$ & $r \cdot$ & 10 & $=$ & $=$ & $=$ & $=$ & ri & va & ro & 17 & 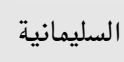 \\
\hline r & rrt & $\leqslant 9$ & 9. & .9 & or & 19 & r & r & $T \varepsilon$ & ir & ro & $=$ & $=$ & $=$ & $=$ & $\cdot \wedge$ & 09 & $1 r$ & 10 & .7 & $\{\Lambda$ & .0 & 19 & بغداد \\
\hline 71 & rro & Tז & 07 & 11 & $\leqslant 9$ & rI & 11 & $=$ & $=$ & $=$ & $=$ & $r$. & 71 & Ir & 11 & $1 \varepsilon$ & 01 & IV & $\cdot 9$ & .9 & $V \varepsilon$ & ir & rI & القادسية \\
\hline VY & TrV & rr & $\mu_{\Lambda}$ & $=$ & $=$ & $=$ & $=$ & $1 \varepsilon$ & r & .0 & 10 & r & $0\}$ & $\cdot 1$ & $1 \varepsilon$ & $r$. & 70 & 11 & .7 & 10 & $\{0$ & $\cdot v$ & r & الموصل \\
\hline 09 & YOT,S & $0 \leqslant, T$ & $T \cdot, Y$ & & & & & & & & & & & & & & & & & & & & & \\
\hline
\end{tabular}


جدول (r) يبين النسب المئوية لمركبات الهجوم المؤثرة

\begin{tabular}{|c|c|c|c|c|c|c|c|c|c|c|c|c|c|c|c|c|c|c|c|c|c|c|c|c|}
\hline \multicolumn{4}{|c|}{ المحاولات الكلية } & \multicolumn{4}{|c|}{ الموصل } & \multicolumn{4}{|c|}{ القادسية } & \multicolumn{4}{|c|}{ بغداد } & \multicolumn{4}{|c|}{ السليمانية } & \multicolumn{4}{|c|}{ صلاح الدين } & \multirow{2}{*}{ المركبات } \\
\hline 变 & $\frac{9}{3}$ & 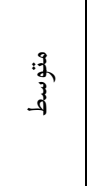 & 紊 & 衰 & $\frac{9}{3}$ & $\frac{3}{3}$ & $\frac{1}{3}$ & $\overline{3}$ & $\frac{9}{3}$ & 3 & 亨 & 豙 & $\frac{9}{3}$ & 3 & 高 & $\overline{3}$ & $\frac{9}{3}$ & 年 & 京 & $\overline{3}$ & $\begin{array}{l}\frac{9}{3} \\
\bar{z} \\
\frac{1}{3}\end{array}$ & 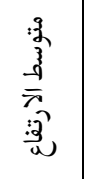 & $\begin{array}{l}\overline{\bar{a}} \\
\overline{\overline{3}} \\
3\end{array}$ & \\
\hline 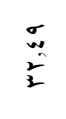 & 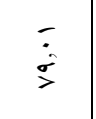 & $\stackrel{\%}{*}$ & 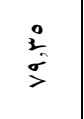 & 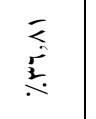 & 亲 & $\vdots$ & 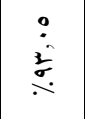 & 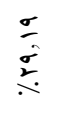 & 衰 & 喜 & $\frac{5}{5}$ & 点 & 常 & 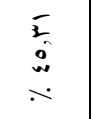 & 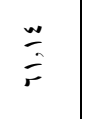 & 立 & 产 & 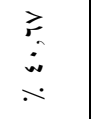 & 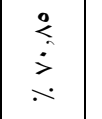 & $=$ & $=$ & $=$ & $=$ & صلاح الدين \\
\hline 点 & 总 & 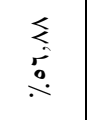 & 登 & 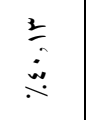 & 紊 & 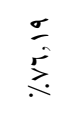 & 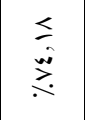 & 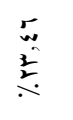 & 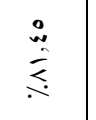 & $\stackrel{3}{\grave{c}}$ & 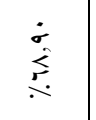 & 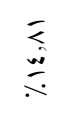 & 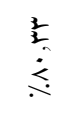 & 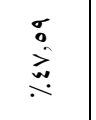 & 年 & $=$ & $=$ & $=$ & $=$ & 意 & 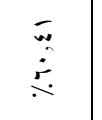 & 总 & 年 & السليمانية \\
\hline$\grave{i}$ & $\frac{5}{5}$ & 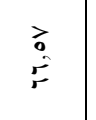 & 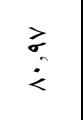 & $\stackrel{\varrho}{\varrho}$ & 旁 & 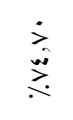 & $\stackrel{?}{:}$ & $\dddot{z}$ & 菊 & 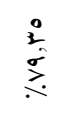 & 紊 & $=$ & $=$ & $=$ & $=$ & 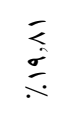 & $\begin{array}{l}\bar{\alpha} \\
\vdots \\
\vdots\end{array}$ & 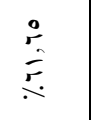 & 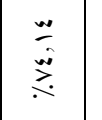 & 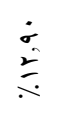 & 意 & 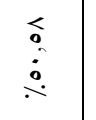 & 妾 & بغداد \\
\hline$\frac{5}{2}$ & 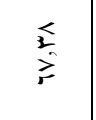 & 旁 & 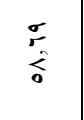 & 亲 & 亲 & 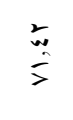 & $\frac{5}{5}$ & $=$ & $=$ & $=$ & $=$ & 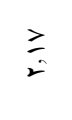 & 言 & 产 & 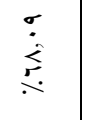 & $\underline{\underline{s}}$ & $\frac{2}{\vdots}$ & 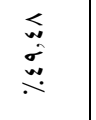 & $\overline{\bar{E}}$ & 童 & $\stackrel{2}{\geq}$ & 竞 & 辛 & القادسية \\
\hline 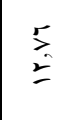 & $\overline{\dot{b}}$ & $\underline{\underline{z}}$ & 范 & $=$ & $=$ & $=$ & $=$ & 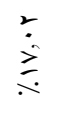 & 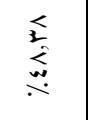 & $\vdots$ & 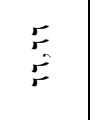 & 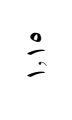 & 㒸 & 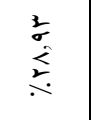 & 竧 & $\bar{I}$ & 紊 & 亲 & 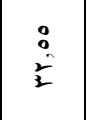 & 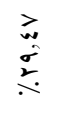 & 旁 & 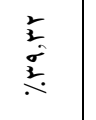 & 产 & الموصل \\
\hline 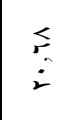 & 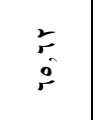 & $\grave{\vdots}$ & 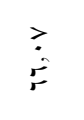 & & & & & & & & & & & & & & & & & & & & & \\
\hline
\end{tabular}




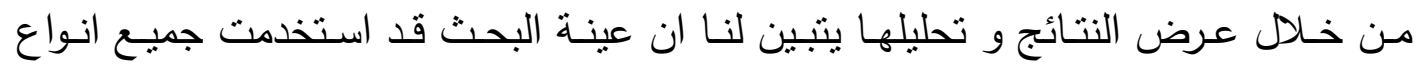

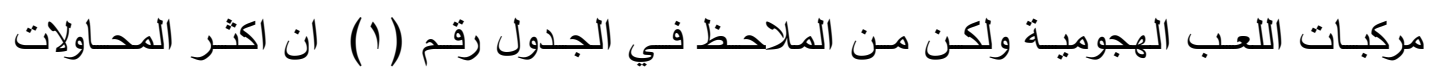

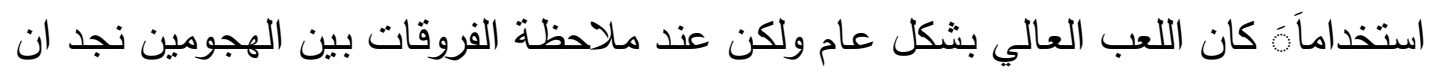

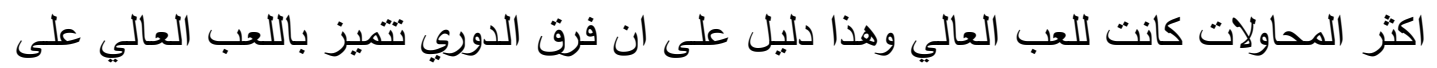
الاكثر وهذا لايشمل فقط عدد المحاولات وانما كان لهذين النوعين من المركبات نأثيراً كبيراً

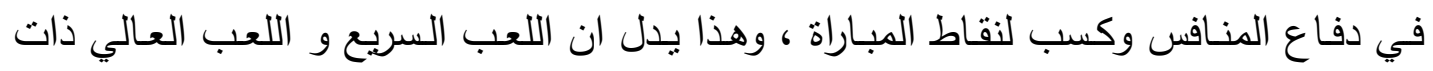
تأثير جيد يدعم نتيجة الفريق من جهة ومن جهة اخرى يعزو الباحث سبب ذلك الى ان مدربي

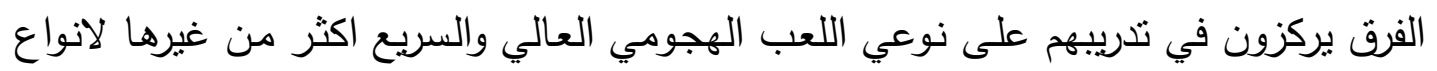

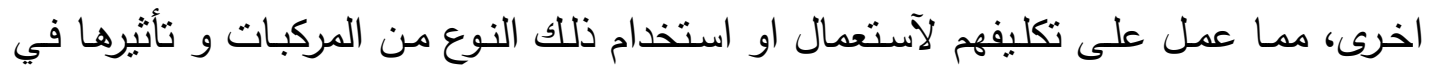
حائط المنافس ، ولكن برجع سبب التركيز في اللعب العالي والسريع عند فرق الجامعات

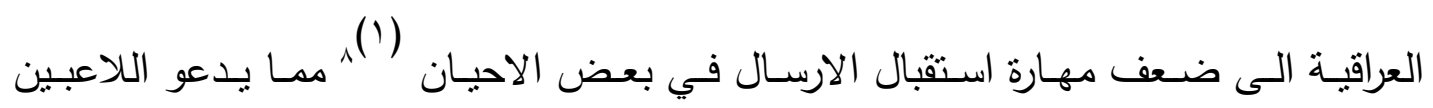

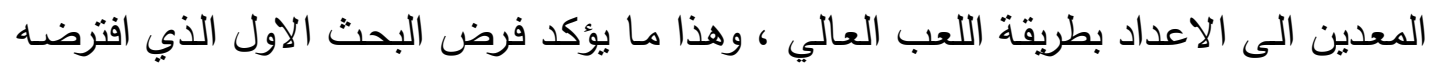

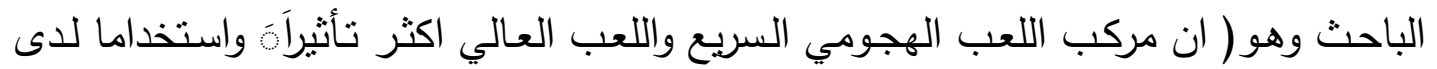

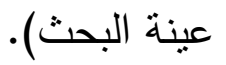

وعند ملاحظة الجدول رقم (r) نجد ان اكثر المركبات استخداماًة و تأثثراًة هي اللعب

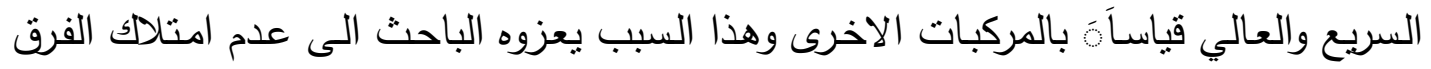
لاعبين جيدين متخصصين بالضرب في المنطقة الخلفية ومتوسط الارتفاع وعدم امتلاكهم المهارات والنواحي البدنية ومنها قوة القفز فضلاًة عن القياسات الجسمية الجيدة الكلي وهذه

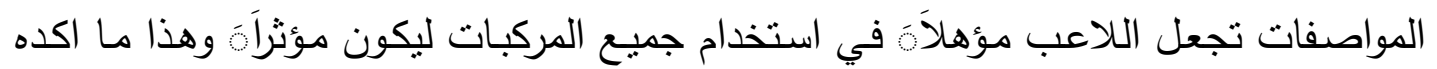
فرض الباحث الثاني (ان مركب اللعب الهجومي في المنطقة الخلفية و متوسط الارتفاع اقل

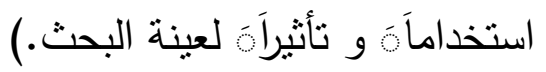

(') عامر جبار السعدى : مهارة استقبال الارسال واثرها في النهج الهجومي ، رسالة ماجستير ، جامعة بغداد ،

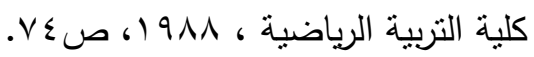




\section{0- الاستنتاجات و التوصيات}

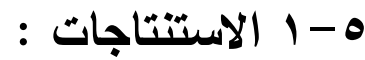

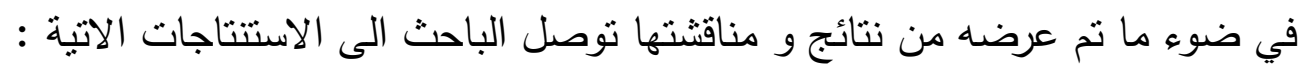

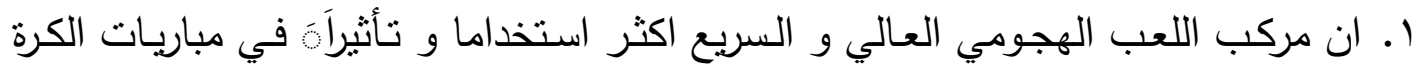

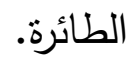

r. ان مركب اللعب الهجومي في المنطقة الخلفية واللعب المنوسط الارتفاع اقل استخداما و

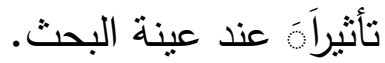

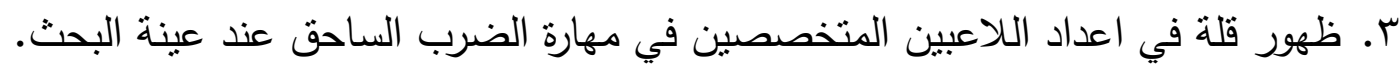

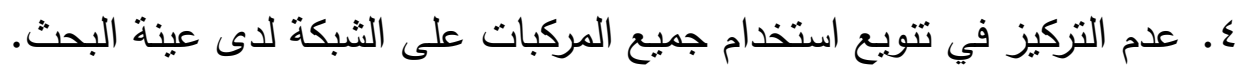

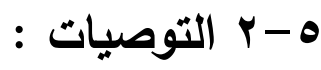

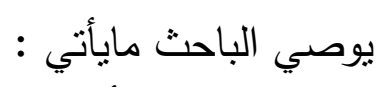

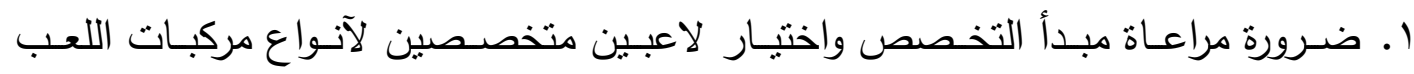
الهجومي على الثبكة.

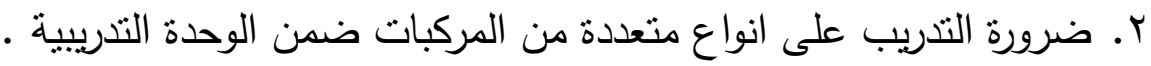

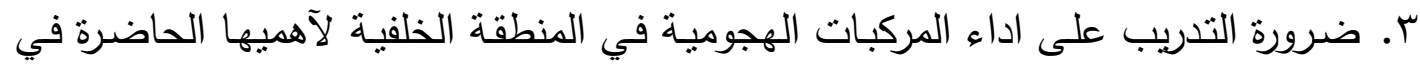
مباريات الكرة الطائرة. ع. ضرورة البحث في انواع الاعداد للمركبات الهجومية المستخدمة لدى عينة البحث. المصادر العربية والأجنبية

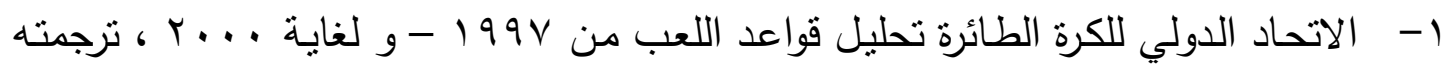

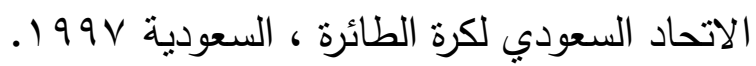

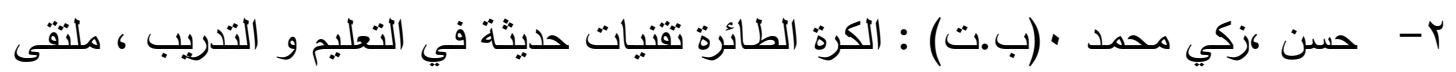

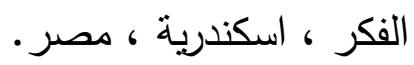
ץ- السعدى ،عامر جبار .(911 (1): مهارة استقبال الارسال واثرها في النهج الهجومي ، رسالة ماجستير ، جامعة بغداد ، كلية التربية الرياضية.

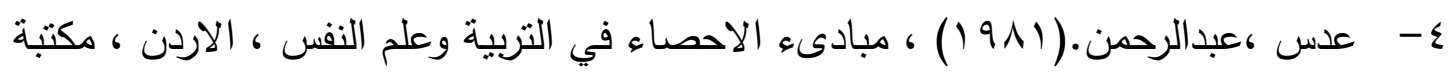

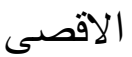
0- قادر ، ازاد حسن.(911 (1) : دراسـة مقارنـة لتأثنير مركبات اللعب الهجومي في المنطقة

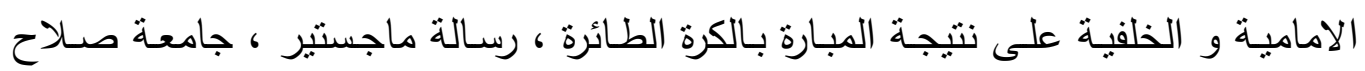
الدين ، كلية التربية الرياضية. 7- الكاتب ،عقيل عبدالله.(9Av (1) ، الكرة الطائرة ، التكنيك والتكنيك الفردي ، بغداد مطبعة

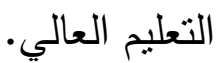

7- Back, horst, Technical report federation international volleyball, 1988 
لملحق رقم (1)

استمارة ملاحظة مركبات اللعب الهجومي في الكرة الطائرة

$$
\begin{aligned}
& \text { اسم الفريق المقابل: } \\
& \text { الملعب : }
\end{aligned}
$$

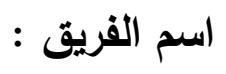$$
\text { التاريخ : }
$$

\begin{tabular}{|c|c|c|c|c|c|c|c|c|c|c|c|c|c|c|c|c|}
\hline \multicolumn{16}{|c|}{ درجات التقييم } & \multirow{3}{*}{ الأتشواط } \\
\hline \multicolumn{4}{|c|}{ في المنطقة الخلفية } & \multicolumn{4}{|c|}{ عالية الارتفاع } & \multicolumn{4}{|c|}{ متوسط الارتفاع } & \multicolumn{4}{|c|}{ اللعب السريع } & \\
\hline 3 & 2 & 1 & 0 & 3 & 2 & 1 & 0 & 3 & 2 & 1 & 0 & 3 & 2 & 1 & 0 & \\
\hline & & & & & & & & & & & & & & & & 1 \\
\hline & & & & & & & & & & & & & & & & $r$ \\
\hline & & & & & & & & & & & & & & & & $r$ \\
\hline & & & & & & & & & & & & & & & & $\varepsilon$ \\
\hline & & & & & & & & & & & & & & & & 0 \\
\hline & & & & & & & & & & & & & & & & المجموع \\
\hline
\end{tabular}$$
\text { رقم المباراة : }
$$ 